\title{
Comparison of RP, lon-Pair and IE Chromatography Coupled with UV and Tandem Mass Spectrometry for the Determination of 8-Hydroxyquinoline in Cosmetics
}

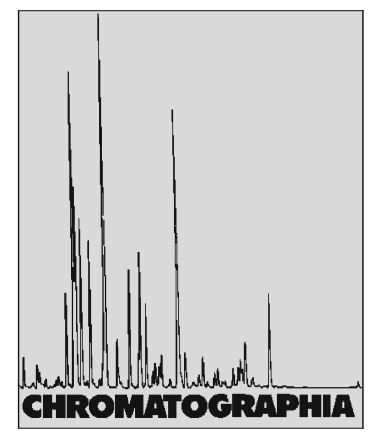

2010, 71, 469-474

\author{
Qiang Ma ${ }^{1,2, 凶}$, Chao Wang ${ }^{1}$, Hua Bai ${ }^{1}$, Wei Ma ${ }^{3,4}$, Xin Zhou' ${ }^{1}$ Yiyang Dong ${ }^{1}$, Qing Zhang ${ }^{1}$ \\ ${ }^{1}$ Chinese Academy of Inspection and Quarantine, 100123 Beijing, China; E-Mail: maqiang1 129@yahoo.com.cn \\ 2 State Key Laboratory of Environmental Chemistry and Ecotoxicology, Research Center for Eco-Environmental Sciences, \\ Chinese Academy of Sciences, 100085 Beijing, China \\ ${ }^{3}$ China Agriculture University, 100083 Beijing, China \\ ${ }^{4}$ Heilongiiang Entry-Exit Inspection and Quarantine Bureau, 150086 Harbin, China
}

Received: 11 September 2009 / Revised: 2 November 2009 / Accepted: 1 December 2009

Online publication: 8 January 2010

\begin{abstract}
Liquid chromatographic methods based on reversed-phase, ion-pair and ion-exchange chromatography coupled with ultraviolet spectrometry and tandem mass spectrometry have been developed and compared for the determination of 8-hydroxyquinoline in cosmetic samples. The individual parameters of different techniques are systematically optimized. The reversed-phase chromatography using ultrasonic extraction, centrifugation and filtration prior to LC-UV and LC-MS-MS detection was selected to be the general analytical method for the routine analysis of 8-hydroxyquinoline in cosmetics due to its simplicity, column reproducibility and sensitivity. The procedure is rapid, simple and suitable for the quality control of cosmetic preparations. The application of this technique to the assay of commercial products is also presented.
\end{abstract}

\section{Keywords}

Column liquid chromatography

lon-pair chromatography

lon-exchange chromatography

8-Hydroxyquinoline in cosmetics

\section{Introduction}

8-Hydroxyquinoline, known as a monoprotic bidentate chelating agent, is extensively used as the antiseptic, disinfectant and deodorant in the production and preservation of cosmetic products for its strong antimicrobial property [1]. 8-Hydroxyquinoline has demonstrated some oncogenic potential when implanted in the mouse bladder in a cholesterol vehicle and when instilled in the rat vagina $[2,3]$. Since cosmetics are widely used consumer goods, the related regulations, as their prime and principal purpose, highly concern the guaranteeing of consumer safety. According to the European Union Council Directive 76/768/EEC, 8-hydroxyquinoline is listed as the substance which must not form part of the composition of cosmetic products except for the uses under maximum authorized concentration restrictions of $0.3 \%(w / w)$ in rinse-off hair-care preparations and $0.03 \%(w / w)$ in non-rinse-off hair-care preparations [4]. Hence, the assay of 8-hydroxyquinoline in commercial cosmetic products is important for quality control purposes and for checking their conformance to the current legislation.

The widely used methods for the determination of 8-hydroxyquinoline are titrimetry [5], polargraphy [6], colorimetry [7, 8], spectrophotometry [9], thin layer chromatography (TLC) [10], and liquid chromatography (LC) [11-13]. However, a detailed study of these publications would indicate that improvement is in great need, since there are no analytical methods specifically for the analysis of 8-hydroxyquinoline in cosmetics so far. It is also necessary to provide the sample pretreatment and 
instrumentation protocol with greater specificity and sensitivity.

In this study, the analytical methods based on reversed-phase, ion-pair and ion-exchange chromatography coupled with ultraviolet spectrometry and tandem mass spectrometry were established, and the chromatographic behaviors in different liquid chromatographic modes were compared. The proposed methods have been successfully applied to determine the content of 8-hydroxyquinoline in cosmetic products, and is applicable to the quality control process of cosmetics to verify their adherence to relevant legislation.

\section{Experimental}

\section{Reagents and Materials}

Organic solvents such as methanol, acetonitrile and tetrahydrofuran of LC grade were from Fisher (Pittsburgh, PA, USA). 8-Hydroxyquinoline of $99 \%$ purity was obtained from Chem Service (West Chester, PA, USA). Sodium heptyl sulfate was from J.T. Baker (Phillipsburg, NJ, USA). Sodium hexyl sulfate and sodium decyl sulfate were purchased from Dikma (Lake Forest, CA, USA). Trifluoroacetic acid (TFA) was from Tedia (Fairfield, OH, USA), phosphoric acid and potassium dihydrogen phosphate were obtained from Alfa Aesar (Ward Hill, MA, USA). All water used in this study was prepared with a Milli-Q water purification system (Millipore, Bedford, MA, USA) under a resistivity of $18.2 \mathrm{M} \Omega \mathrm{cm}^{-1}$. The $\mathrm{pH}$ meter was from Mettler Toledo (Columbus, OH, USA).

\section{Sample Collection and Preparation}

A variety of untreated cosmetic samples of different categories (lotion, cream, liquid, gel, powder, shampoo, etc.) were collected from the cosmetic industries of China, France, United State, Germany, Italy, Japan, Korea, etc. The origins of all of the cosmetic samples were known before analysis. About $0.5 \mathrm{~g}$ of the cosmetic sample was placed into a glass tube, and $10 \mathrm{~mL}$ of methanol was added. The glass tube was shaken on a vortex mixer for about $30 \mathrm{~s}$, and then extracted under sonication for $20 \mathrm{~min}$. After centrifuged at $12,000 \mathrm{rpm}$ for $10 \mathrm{~min}$, the clear supernatant was filtered by $0.45 \mu \mathrm{m}$ membrane before injection for instrumental analysis.

\section{Instrument and Chromatography}

Liquid chromatography was performed on a 1200 LC system (Agilent Technologies, Palo Alto, CA, USA) equipped with online degasser, quaternary pump, autosampler with $100 \mu \mathrm{L}$ injection loop, thermostatic compartment, and diode array detector. Analysis of 8-hydroxyquinoline was carried out based on different liquid chromatographic separation mechanism, i.e. reversed-phase, ion-pair and ion-exchange chromatography respectively, with the detection wavelength of $240 \mathrm{~nm}$ for optimum sensitivity.

The separation of 8-hydroxyquinoline in the reversed-phase chromatography was conducted with a Waters XTerra MS $\mathrm{C}_{18}$ chromatographic column $(4.6 \mathrm{~mm}$ id $\times 250 \mathrm{~mm}$ length, $5 \mu \mathrm{m}$ particle size). The mobile phase for eluting the analyte comprised 20\% A $(0.1 \%$ trifluoroacetic acid in methanol) and $80 \% \mathrm{~B}(0.1 \%$ trifluoroacetic acid in

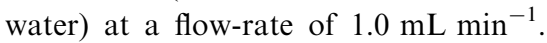
The ion-pair chromatography was performed on a chromatographic column packed with Kromasil $\mathrm{C}_{18} \quad(4.6 \mathrm{~mm}$ id $\times 250 \mathrm{~mm}$ length, $5 \mu \mathrm{m}$ particle size) under isocratic elution with the mobile phase of $55 \%$ A (consisting of $10 \mathrm{mmol}$ $\mathrm{L}^{-1}$ potassium dihydrogen phosphate and $20 \mathrm{mmol} \mathrm{L}^{-1}$ sodium heptyl sulfate, adjusted to $\mathrm{pH} 2.25$ with orthophosphoric acid) and 45\% $\mathrm{B}$ (methanol) at a flow-rate of $1.0 \mathrm{~mL} \mathrm{~min}{ }^{-1}$. The ionexchange chromatography was carried out on a Waters Spherisorb SCX chromatographic column $(4.6 \mathrm{~mm}$ id $\times$ $250 \mathrm{~mm}$ length, $5 \mu \mathrm{m}$ particle size) under isocratic elution with the mobile phase composed of $55 \%$ A $\left(150 \mathrm{mmol} \mathrm{L} \mathrm{L}^{-1}\right.$ potassium dihydrogen phosphate, adjusted to $\mathrm{pH} 2.25$ with orthophosphoric acid) and 45\% $\mathrm{B}$ (methanol) at a flow-rate of $1.0 \mathrm{~mL} \mathrm{~min}^{-1}$. In all the three methods, the column was thermostated at $35^{\circ} \mathrm{C}$ and the UV detection at wavelength $240 \mathrm{~nm}$ was used for the determination of 8-hydroxyquinoline. The chromatographic parameters of 8-hydroxyquinoline in the reversed-phase, ion-pair and ion-exchange chromatography are shown in Table 1.

\section{Mass Spectrometry}

A Quattro micro atmospheric pressure ionization (API) mass spectrometer (Micromass, Manchester, UK) equipped with an ESI source was used. The Z-Spray dual orthogonal sampling interface allowed the entire column effluent from the liquid chromatograph to be directed into the MS source without flow splitting, which contributed to the greatly enhanced sensitivity and ruggedness. Typical ion source parameters were set as follows: ESI capillary voltage $3.5 \mathrm{kV}$, cone voltage $35 \mathrm{~V}$, extractor voltage $5 \mathrm{~V}$, RF lens voltage $0.5 \mathrm{~V}$, source block temperature $120^{\circ} \mathrm{C}$, desolvation temperature $350{ }^{\circ} \mathrm{C}$, nitrogen desolvation gas flow rate $500 \mathrm{~L} \mathrm{~h}^{-1}$, cone gas flow rate $50 \mathrm{~L} \mathrm{~h}^{-1}$, ion energy 1 and $20.5 \mathrm{~V}$, and multiplier voltage $650 \mathrm{~V}$. On the occasion of the presence of 8-hydroxyquinoline in the real cosmetic samples detected by liquid chromatography, it was necessary to conduct LC-MS-MS analysis for confirmation. Qualitative analysis was performed in multiple reaction monitoring (MRM) mode under positive ion conditions, in which the analyte was first detected as protonated molecular ion of 145.9, and then fragmented in the MS-MS mode to produce the product ions of 117.6 and 127.7 with the collision energy of 18 and $23 \mathrm{eV}$, respectively, to achieve four identification points complying with the European Union Directive 2002/657/EC [14].

\section{Results and Discussion}

\section{Silanol Effect}

8-Hydroxyquinoline is an amphoteric compound containing two ionizable functional groups individually capable 
Table 1. Chromatographic parameters for 8-hydroxyquinoline in the reversed-phase chromatography, ion-pair chromatography and ionexchange chromatography

\begin{tabular}{|c|c|c|c|}
\hline Parameters & $\begin{array}{l}\text { Reversed-phase } \\
\text { chromatography }\end{array}$ & $\begin{array}{l}\text { Ion-pair } \\
\text { chromatography }\end{array}$ & $\begin{array}{l}\text { Ion-exchange } \\
\text { chromatography }\end{array}$ \\
\hline Chromatographic column & $\begin{array}{l}\text { XTerra MS C }{ }_{18} \text { column } \\
\quad(250 \mathrm{~mm} \times 4.6 \mathrm{~mm}, 5 \mu \mathrm{m})\end{array}$ & $\begin{array}{l}\text { Kromasil } \mathrm{C}_{18} \text { column } \\
\quad(250 \mathrm{~mm} \times 4.6 \mathrm{~mm}, 5 \mu \mathrm{m})\end{array}$ & $\begin{array}{l}\text { Spherisorb SCX column } \\
\quad(250 \mathrm{~mm} \times 4.6 \mathrm{~mm}, 5 \mu \mathrm{m})\end{array}$ \\
\hline Column temperature $\left({ }^{\circ} \mathrm{C}\right)$ & 30 & 30 & 30 \\
\hline Mobile phase & $\begin{array}{l}20 \% \text { A }(0.1 \% \text { TFA in } \\
\text { methanol }) \text { and } 80 \% \\
\text { B }(0.1 \% \text { TFA in water })\end{array}$ & $\begin{array}{l}55 \% \text { A }\left(10 \mathrm{mmol} \mathrm{L}^{-1} \text { potassium }\right. \\
\text { dihydrogen phosphate }+20 \mathrm{mmol} \mathrm{L} \\
\text { sodium heptyl sulfate, } \mathrm{pH} 2.25 \\
\text { adjusted with orthophosphoric acid) } \\
\text { and } 45 \% \mathrm{~B} \text { (methanol) }\end{array}$ & $\begin{array}{l}55 \% \text { A }\left(150 \mathrm{mmol} \mathrm{L}^{-1}\right. \\
\text { potassium dihydrogen } \\
\text { phosphate, pH } 2.25 \text { adjusted } \\
\text { with orthophosphoric acid) } \\
\text { and } 45 \% \mathrm{~B} \text { (methanol) }\end{array}$ \\
\hline Flow rate $\left(\mathrm{mL} \min ^{-1}\right)$ & 1.0 & 1.0 & 1.0 \\
\hline Detection wavelength (nm) & 240 & 240 & 240 \\
\hline Injection volume $(\mu \mathrm{L})$ & 10 & 10 & 10 \\
\hline
\end{tabular}

of acidic or basic behavior with the dissociation constants of $\mathrm{p} K_{1}=5.0$ and $\mathrm{p} K_{2}=9.9$, respectively $[15,16]$. The chromatography concerning the amphoteric compound is very complicated due to several concurrent retention mechanisms which may possibly govern the chromatographic behavior simultaneously, including ion-exchange, hydrophobic interaction, hydrogen-bond interaction, etc. 8-Hydroxyquinoline, as an ionic compound, may interact with the residual silanols inside the silicabased columns in liquid chromatography, and therefore lead to the phenomena of excessive retention, severe band tailing and column-to-column irreproducibility because of silanophilic interactions. It is generally desirable to minimize the silanol interaction effect by adopting appropriate experimental conditions, such as adjusting mobile-phase $\mathrm{pH}$ to restrain residue silanols activity or creating the environment containing ion-pair agent to control silanol effect.

\section{Optimization of the Reversed-Phase Chromatographic Parameters}

In reversed-phase chromatography, the resulting surface charge of stationary phase mainly depends on the number of positively charged sites and the number of negatively charged sites and is therefore a function of solution $\mathrm{pH}$. The influence of silanol group on the chromatographic behavior of the sample may be suppressed either by using a low-pH mobile phase to minimize the concentration of ionized silanols on the column surface and to reduce the ion-exchange interactions with the solute or by using a high-pH mobile phase to keep the analyte in an uncharged form and to inhibit the ion-exchange ability of residual silanols toward the protonated analyte. As a result, the selection of proper buffer $\mathrm{pH}$ is necessary to reproducibly separate ionizable compounds to achieve a sharp and symmetrical peak. In this study, the $\mathrm{pH}$ ranges from 1.5 to 11.0 with a $\mathrm{pH}-$ stable column and a series of buffer solutions corresponding to the target pH: pH 1.5 (0.1\% trifluoroacetic acid $)$; $\mathrm{pH} 2.0,2.25$ and $3.0\left(10 \mathrm{mmol} \mathrm{L}^{-1}\right.$ potassium dihydrogen phosphate); $\mathrm{pH}$ 3.5 , 4.0 and $4.5\left(10 \mathrm{mmol} \mathrm{L}^{-1}\right.$ ammonium formate); $\mathrm{pH} \quad 5.0$ and 5.5 (10 mmol $\mathrm{L}^{-1}$ ammonium acetate); $\mathrm{pH}$ $6.0,6.5,7.0,7.5$ and $8.0\left(10 \mathrm{mmol} \mathrm{L}^{-1}\right.$ potassium hydrogen phosphate); $\mathrm{pH} 9.0$, 10.0 and $11.0\left(10 \mathrm{mmol} \mathrm{L}^{-1}\right.$ ammonium bicarbonate) were investigated. The mobile-phase $\mathrm{pH}$ was adjusted to the target $\mathrm{pH}$ before adding organic solvent. The results showed that $\mathrm{pH}$ was critical for 8-hydroxyquinoline separation and the best chromatographic peak shape and acceptable retention was found at $\mathrm{pH}$ 2.25. On the other hand, the chromatographic performance at high $\mathrm{pH}$ was far from satisfactory and resulted in bad peak shape. Choice of chromatographic column type including $\mathrm{C}_{18}, \mathrm{C}_{8}$, phenyl, cyano, etc., was studied in order to obtain the optimum performance. Excellent result was exhibited by using a Waters XTerra MS $\mathrm{C}_{18}$ chromato- graphic column $(4.6 \mathrm{~mm}$ id $\times 250 \mathrm{~mm}$ length, $5 \mu \mathrm{m}$ particle size). In terms of solvent type, the three most widely used organic mobile phase, i.e. methanol, acetonitrile and tetrahydrofuran, were observed to show similar chromatographic performance in respective proper proportion to aqueous mobile phase. Methanol was finally chosen to be the first choice because of its relatively lower toxicity, price and greater compatibility for buffers compared to mobile phase containing acetonitrile or tetrahydrofuran. The volume fraction of methanol in the aqueous-organic mobile phase was optimized to obtain the suitable capacity factor under isocratic conditions in a binary mobile phase. It was reported in some literatures [17, 18] that the addition of amine modifiers is beneficial for the chromatographic separation of basic samples due to the blockage of ionized silanols by the amine. In our study, triethylamine and hexylamine, as the common amine modifiers, were tested to observe whether there was even better performance. However, no improved chromatographic behavior of 8-hydroxyquinoline was found. Furthermore, amine modifiers were out of consideration because they can cause slow column equilibration when changing the mobile phase. Silanol effect may also be reduced by choosing a buffer cation which can be held more strongly by the silanols than the analyte and therefore block unnecessary sample retention by the ionized silanols. Buffers in the potassium form normally have strong affinity to silanols and good 
solubility in aqueous-organic mobile phase. Taking into account these facts, potassium dihydrogen phosphate was finally chosen to be used in our system, and different buffer concentrations in the mobile phase were optimized. Temperature generally has a much more noticeable effect for the reversed-phase separation of ionic samples than neutral samples, since several retention-related interactions can be involved, each corresponding differently to the changes in temperature. A column temperature of $30{ }^{\circ} \mathrm{C}$ was optimized for sample analysis.

\section{Optimization of the lon-Pair Chromatographic Parameters}

In the ion-pair chromatographic mode for the analysis of 8-hydroxyquinoline, the negative ion-pair reagent added is attached to the stationary phase by hydrophobic interaction with alkyl moiety, leaving the stationary phase carried by the negative charged group (e.g., $\mathrm{C}_{9}-\mathrm{SO}_{3}{ }^{-}$), which is further balanced by positive ions (e.g., $\mathrm{Na}^{+}$) from the ionpair reagent and positive ions (e.g., $\mathrm{K}^{+}$) from the buffer. The protonated and positively charged 8-hydroxyquinoline can now exchange with the above $\mathrm{Na}^{+}$and $\mathrm{K}^{+}$ions, resulting in the retention of 8-hydroxyquinoline by an ion-exchange process. A series of chromatographic parameters, such as ionpair reagent type and concentration, $\mathrm{pH}$ value, buffer type and concentration, solvent type and strength, column temperature were evaluated. In view of the basic property of 8-hydroxyquinoline, several commonly used negative ion-pair reagents, i.e., sodium hexyl sulfate, sodium heptyl sulfate, sodium decyl sulfate were investigated. Sodium heptyl sulfate was shown to have relatively higher efficiency in decreasing solute silanophilic interactions and relatively shorter column equilibration duration due to its moderate hydrophobicity. The presence of sodium heptyl sulfate as the ion-pair reagent significantly increased the retention time of 8-hydroxyquinoline. The mobile phase contains a large number of negatively charged species $\left(\mathrm{SO}_{3}{ }^{-}\right)$which compete for the analyte and block active, strongly interacting sites on the surface of silica. A reasonable sodium heptyl sulfate concentration of $20 \mathrm{mmol} \mathrm{L}^{-1}$ was chosen in the mobile phase according to retention versus reagent concentration curve to achieve the significant retention of the analyte on the column. In the case of the interaction between 8-hydroxyquinoline and sodium heptyl sulfate, low $\mathrm{pH}$ is preferable due to the occurrence of maximum retention as well as the contribution to silanol effect suppression. A pH of 2.25 was found to be the optimum and $10 \mathrm{mmol} \mathrm{L}^{-1}$ potassium dihydrogen phosphate buffer with proper buffering range was correspondingly adopted. It is rather important to maintain a constant $30{ }^{\circ} \mathrm{C}$ column temperature by thermostat compartment to stabilize several equilibrium processes, such as sample retention by ion-exchange, sorption of the ion-pair reagent on the chromatographic column, reversed-phase processes, etc. When ion-pair chromatography was used, the column was conditioned by passing the mobile phase through it for at least $2 \mathrm{~h}$ at a flow-rate of $1 \mathrm{~mL} \mathrm{~min}^{-1}$.

\section{Optimization of the lon- Exchange Chromatographic Parameters}

Ion-exchange is one of the important retention mechanisms for amphoteric compounds in liquid chromatography. Since there is much similarity between ion-exchange and ion-pair chromatography in terms of retention behavior, many separations that are possibly using ion-exchange chromatography can also be achieved using ion-pair chromatography. In the current study, a cationexchange column with negative sulfonate group covalently embedded in the stationary phase is utilized for the assay of 8-hydroxyquinoline. Various chromatographic parameters, including buffer type and concentration, column temperature, mobile phase composition, etc., were optimized in the similar manner to reversed-phase chromatographic and ion-pair chromatographic analysis of 8-hydroxyquinoline.

\section{Comparison of the Reversed- Phase, lon-Pair and lon- Exchange Chromatography}

Chromatographic parameters of reversed-phase chromatography, ion-pair chromatography and ion-exchange chromatography were summarized in Table 1. Results of some typical system suitability data in the three chromatographic modes are listed in Table 2.

In the reversed-phase, ion-pair as well as ion-exchange chromatography, acceptable retention of 8-hydroxyquinoline can be achieved by adjusting the chromatographic parameters. Under the preconditions that all the chromatographic columns utilized in the three chromatographic modes were newly coming into use and were specifically for the analysis of 8-hydroxyquinoline, ionexchange chromatography exhibited the best peak symmetry, i.e. highest symmetry factor and lowest USP tailing factor. Ion-pair chromatography, in terms of column efficiency for the assay of 8-hydroxyquinoline, was shown to possess the best performance. And reversed-phase chromatography obtained the best repeatability according to the data of RSD of peak area and retention time of 8-hydroxyquinoline. As a result, there is no such a chromatographic mode that has shown dominating advantages over the others and each of them has their own features in certain aspects.

For regular ionic samples, we have a choice of three LC methods: reversedphase, ion-pair, or ion-exchange chromatography. Because of its simplicity, freedom from problems, and better column reproducibility, reversed-phase chromatography usually is the best starting point for the analysis. Ion-pair chromatography is a logical follow-up to reversed-phase chromatography for the separation of ionic samples, differing mainly in the addition of an ion-pair reagent to the mobile phase. Ionexchange chromatography is also an alternative method for the analysis of basic compounds. Reversed-phase chromatography and ion-exchange chromatography with a volatile buffer may be compatible with a mass spectrometer 
detector, whereas the ion-pair reagent used in this study for ion-pair chromatography is not sufficiently volatile and is not applicable to liquid chromatography coupled with mass spectrometer detection. Generally speaking, reversed-phase chromatography was selected to be the general method for the routine analysis of 8-hydroxyquinoline of the three chromatographic methods, possessing the advantages of column performance, operation simplicity, lower cost, MS compatibility, etc.

\section{Optimization of LC-MS-MS Parameters}

Given that non-volatile salt should be avoided and the volatile additive concentration should be kept low enough in order to prevent ion source contamination, ammonium acetate was finally chosen in our system. A series of mass spectrometric parameters, including ESI capillary voltage, cone voltage, extractor voltage, RF lens voltage, collision energy, etc., were optimized detailedly. For the detection by LC-MS-MS, identification occurs with the retention time and diagnostic ions consisting of the daughter ions at $m / z 117.6$ and 127.7. For confirmatory purpose, a comparison with quality control samples is made using acceptable deviations of $\pm 2.5 \%$ for retention time and of $\pm 20 \%$ for the ionic relative abundance as described in the European Commission Directive 2002/657/EC laying down performance criteria of analytical methods.

\section{Method Validation}

The established reversed-phase chromatographic method was validated for recovery, limit of detection (LOD), linear dynamic range, precision and reproducibility. Recoveries of spiked compound at three levels were between 93.2 and $100.8 \%$. LOD based on injection of a $10 \mu \mathrm{L}$ aliquot, calculated as a signal-to-noise $(S / N)$ ratio of three, was $1 \mathrm{ng}$. The linear dynamic range of the reversed-phase chromatographic instrumentation was evaluated by analyzing 8-hydroxyquinoline standard solutions

Table 2. System suitability data for 8 -hydroxyquinoline in the reversed-phase, ion-pair and ionexchange chromatography

\begin{tabular}{|llll|}
\hline Property & $\begin{array}{l}\text { Reversed-phase } \\
\text { chromatography }\end{array}$ & $\begin{array}{l}\text { Ion-pair } \\
\text { chromatography }\end{array}$ & $\begin{array}{l}\text { Ion-exchange } \\
\text { chromatography }\end{array}$ \\
\hline$t_{\mathrm{R}}(\mathrm{min})$ & 9.275 & 8.607 & 9.502 \\
Capacity factor & 2.508 & 2.255 & 2.594 \\
Symmetry factor & 0.717 & 0.662 & 0.789 \\
USP tailing factor & 1.328 & 1.466 & 1.201 \\
PWHM & 0.197 & 0.160 & 0.189 \\
Plate & 12,334 & 16,001 & 13,960 \\
Repeatability, A (RSD, \%) & 0.62 & 0.97 & 0.85 \\
Repeatability, $t_{\mathrm{R}}(\mathrm{RSD}, \%)$ & 0.02 & 0.05 & 0.04 \\
\hline
\end{tabular}

at the following injected amounts: 1, 5, 10, 50, 100, $500 \mathrm{ng}$. Calibration curve was generated using linear regression analysis and gave good fit, with the correlation coefficients of 0.9999 over the established range. The precision of the method was determined from the extraction and analyses of triplicate samples. Over the concentration range, within- and between-day precision were found to be 2.15 and $3.12 \%$, respectively. The reproducibility of the method was determined from triplicate analyses of spiked cosmetic samples. The overall reproducibility of the analyses was satisfactory, with the relative standard deviation (RSD) value of $3.68 \%$.

\section{Analysis of Cosmetic Samples}

A variety of cosmetic samples of different categories (lotion, cream, liquid, gel, powder, shampoo, etc.) were analyzed by the proposed reversed-phase chromatographic method. From our investigation, there are two hair-care samples found containing 8-hydroxyquinoline with both concentrations lower than $0.03 \%$ of the 30 cosmetic samples tested. The two cosmetic samples were subjected to LC-MS-MS confirmatory test to verify the accuracy of the detection.

\section{Conclusions}

This paper describes and compares a set of LC methods using different techniques, i.e. reversed-phase, ion-pair and ion-exchange chromatography for the determination of 8-hydroxyquinoline in cosmetic samples. The chromatographic parameters in the three modes and
LC-MS-MS parameters were systematically optimized. The reversed-phase chromatography based on ultrasonic extraction, centrifugation and filtration prior to LC-UV and LC-MS-MS was selected to be the general analytical method for the routine screening analyses of 8-hydroxyquinoline in cosmetics due to its simplicity, column reproducibility and sensitivity, and is suitable for the quality control assay of 8-hydroxyquinoline in cosmetic products.

To our knowledge, this is the first report of the analytical method for the determination of 8-hydroxyquinoline in cosmetic samples, and our measurements provide some scientific data about the presence and dosage of 8-hydroxyquinoline in real cosmetic products of different categories and production origins.

\section{Acknowledgements}

This work was supported by the Chinese Entry-Exit Inspection and Quarantine Industrial Standard Foundation (Grant No. 2006B463).

\section{References}

1. Yamamoto RS, Williams GM, Frankel HH, Weisburger JH (1971) Toxicol Appl Pharmacol 19:687-698

2. Boyland E, Watson G (1956) Nature 177:837-838

3. Hueper WC (1965) Arch Pathol 79:245-250

4. Council Directive of 27 July 1976 on the approximation of the laws of the Member States relating to cosmetic products (76/768/EEC)

5. The United States Pharmacopoeia (1995) 23rd Revision, The United States Phar- 
macopoeial Convention, Rockville, MD, pp 2212

6. Mejstrik V, Drzleva L, Sagnes Z (1988) Chem Prum 38:361

7. Artemchenko SS, Petrenko VV (1985) Otkrytiya Izobret 27:144

8. Atemchenko SS, Petrenk VV, Zhovna NP, Tsilinko VA (1985) Anal Khim 40:744

9. Joseph JA, Sezkers L (1976) Talanta 23:558-559. doi:10.1016/0039-9140(76) 80161-0

10. De Kruijf N, Rijk MAH, PranotoSoetardhi LA, Schouten A (1987) J Chromatogr A 410:395-411. doi:10.1016/ S0021-9673(00)90069-4
11. Miura K, Nakamura $H$, Tanaka $H$, Tamura Z (1981) J Chromatogr A 210:536-539. doi:10.1016/S0021-9673(00) 80348-9

12. Nagiel-Ostaszewski I, Vavrek MT, Weisburger JH (1991) Xenobiotica 21:751-754. doi:10.3109/0049825910903 9514

13. Miura K, Nakamura H, Tanaka H, Tamura Z (1979) Chem Pharm Bull 27:1759-1763

14. Commission Decision of 14 August 2002 implementing Council Directive 96/23/EC concerning the performance of analytical methods and the interpretation of results $(2002 / 657 /$ EC $)$
15. Clarke EGC, Moffat AC (1986) Clarke's isolation and identification of drugs, 2nd edn. The Pharmaceutical Press, London, pp 672

16. Weast RC (1980) CRC handbook of chemistry and physics, 61st edn. CRC Press, Boca Raton

17. Smith RM, Rabuor JO (1989) J Chromatogr A 464:117-123. doi:10.1016/S00219673(00)94228-6

18. Vervoort RJM, Maris FA, Hindriks $H$ (1992) J Chromatgr A 623:207-220. doi:10.1016/0021-9673(92)80360-7 